\title{
NONVIRAL TRANSFECTION OF SUSPENSION CELLS IN ULTRASOUND STANDING WAVE FIELDS
}

\author{
Yu-Hsiang LeE* ${ }^{1}$ and ChIng-An PenG ${ }^{\dagger}$ \\ *Department of Chemical Engineering and Materials Science, University of Southern California, Los Angeles, CA; \\ and Department of Chemical Engineering, National Taiwan University, Taipei, Taiwan \\ (Received 19 September 2005, revised 26 October 2006, in final form 31 October 2006)
}

\begin{abstract}
Ultrasound-induced cavitation has been widely used for delivering DNA vectors into cells. However, this approach may seriously disrupt cell membranes and cause lethal damage when cells are exposed to the inertial cavitation field. In this study, instead of using sonoporation, ultrasound standing wave fields (USWF) were explored for nonviral transfection of suspension cells. Acoustic resonance in a tubular chamber was generated from the interference of waves emitted from a piezoelectric transducer and consequently reflected from a borosilicate glass coverslip. The suspended K562 erythroleukemia cells were transfected by polyethyleneimine (PEI)/DNA complexes with and without exposure to 1-MHz USWF for 5 min. During USWF exposure, K562 cells moved to the pressure nodal planes first and formed cell bands by the primary radiation force. Nanometer-sized PEI/DNA complexes, circulated between nodal planes by acoustic microstreaming, then used the cell agglomerates as the nucleating sites on which to attach. After incubation at $37^{\circ} \mathrm{C}$ for $48 \mathrm{~h}$, the efficiency of nonviral transfection based on EGFP transgene expression was determined by fluorescent microscopy and fluorometry. Both studies showed that USWF brought suspended K562 cells and PEI/DNA complexes into close contact at the pressure nodal planes, yielding an approximately 10-fold increment of EGFP transgene expression compared with the group without ultrasonic treatment. (E-mail: chinganpeng@ntu.edu.tw) (C) 2007 World Federation for Ultrasound in Medicine \& Biology.
\end{abstract}

Key Words: Ultrasound standing wave fields, PEI/DNA complex, Transfection, Nonviral gene delivery, Sonoporation.

\section{INTRODUCTION}

Quite a few techniques, classified as viral and nonviral, have been developed to introduce nucleic acids into target cells. The viral approach has been proven to be a very effective method for gene delivery; however, it is plagued by immune response (Marshall 1999) and insertional mutagenesis (Hacein-Bey-Abina et al. 2003). Because of the risky concerns of using viral vectors, a nonviral approach has gradually gained attention, although the achievable transfection efficiency is not as great as using viral vectors. Among reported nonviral techniques such as electroporation, microinjection, lipofection etc. (Luo and Saltzman 2000), acoustically induced transfection has been demonstrated to be a feasible and potential operation to enhance nonviral gene delivery efficiency (Miller et al. 1999). This type of transfec-

Address correspondence to: Ching-An Peng, Department of Chemical Engineering, National Taiwan University, No. 1, Sec. 4, Roosevelt Road, Taipei, 10617, Taiwan. E-mail: chinganpeng@ ntu.edu.tw

${ }^{1}$ Present address: Sierra Sciences LLC, Reno, NV 89502 tion, so-called sonoporation, uses ultrasound to permeabilize cell membrane and concomitantly leads to gene uptake via the diffusion process. Generally speaking, such ultrasound-mediated transfection, substantially governed by acoustic cavitation (Bao et al. 1997; Miller et al. 2002), has been developed into several operation modes including burst ultrasound (Tata et al. 1997), continuous wave ultrasound (Kim et al. 1996; Pislaru et al. 2003), shockwaves (Lauer et al. 1997; Huber et al. 1999) and ultrasound in association with microbubbles (Lawrie et al. 2000; Chen et al. 2003). In addition to its in vitro application to mammalian cells (Fechheimer et al. 1987) and plant cells (Joersbo and Brunstedt 1992), ultrasoundmediated gene delivery has been widely explored for in vivo system (Taniyama et al. 2002; Lu et al. 2003; Miller and Song 2003).

So far, although ultrasound has been demonstrated as a promising tool for gene transfer, the cell membrane may be seriously disrupted and subsequently cause lethal damage by ultrasound-induced physical forces such as shockwaves, shear stresses and microjets (Miller 1987). Judging from the enhancement of retroviral transduc- 
tion efficiency using ultrasound standing wave fields (USWF), reported recently (Lee et al. 2006; Lee and Peng 2006), the effect of USWF on delivering nonviral DNA vectors into cells suspended in three dimensional domain was thereby explored in this study. In the past decade, USWF-mediated cell retention systems have been widely applied to the field of biotechnology, such as filtration, sedimentation, spin filters and centrifugation (Shirgaonkar et al. 2004). The force generated in USWF to drive particles or cells in suspension is the primary radiation force $(F)$

$$
F=\frac{\pi \mathrm{P}_{0}^{2} V \beta_{0}}{2 \lambda} \times \phi(\beta, \rho) \times \operatorname{Sin}\left(\frac{4 \pi z}{\lambda}\right)
$$

where $P_{0}$ is the standing wave peak pressure amplitude, $V$ is the volume of the particle, $\lambda$ is the wavelength of ultrasound and $z$ is the distance in the direction at right angles to the pressure nodal planes. The contrast factor $\phi$ is given by

$$
\phi=\frac{5 \rho_{p}-2 \rho_{0}}{2 \rho_{p}+\rho_{0}}-\frac{\beta_{p}}{\beta_{0}}
$$

where $\rho_{p}$ and $\beta_{p}$ are the density and compressibility of the particle and $\rho_{0}$ and $\beta_{0}$ are the density and compressibility of the surrounding continuous phase, respectively. That is, in a well-defined USWF, primary radiation forces quickly move cells along the direction of sound propagation into areas of vanishing acoustic pressure (i.e., pressure nodes) and thereby achieve the purpose of retention and separation. In view of the unique feature of localizing cells in suspension, USWF was considered as an alternative approach for nonviral gene delivery by increasing the encounter opportunity between nonviral DNA vectors and target cells. During USWF exposure, microparticles (i.e., suspended cells) are driven to the pressure nodal planes by the primary radiation force, which then aggregate to form cell bands separated by half-wavelength intervals (Whitworth and Coakley 1992). Theoretically, suspension cells can arrive at the pressure nodal planes in a time scale of seconds, and the nanoparticles (i.e., nonviral DNA vectors) proceed over a time scale of minutes (Hawkes et al. 1998). However, nanometer-sized DNA vectors do not at a stand still on the pressure nodal planes, but instead of circulating between pressure nodal planes, because microstreaminginduced drag force $(f)$ is comparable with the primary radiation force:

$$
f=-6 \pi \mu R(v-u)
$$

Here, $\mu$ is fluid viscosity, $R$ is particle radius, $v$ is particle velocity and $u$ is microstreaming velocity (Coakley et al. 1989). Interestingly, it was demonstrated that submicrometer-sized bacteria with low concentration could be harvested from suspension if mixed with larger particles such as yeasts (Limaye et al. 1996). In addition, a number of studies indicated that the acoustic fields caused no variations in particle integrity and cell viability in yeasts, mammalian cells or erythrocytes (Doblhoff-Dier et al. 1994; Radel et al. 2000). This observation indicates that smaller particles can be collected by using larger particle clumps aggregated on the pressure nodal planes in USWF as the nucleating sites.

The aim of the present work was to explore USWF instead of cavitation for enhancing nonviral transfection efficiency. Because the binding of DNA vectors onto target cells is crucial for subsequent cellular internalization, we speculate that suspended cells and nonviral DNA vectors can encounter each other at pressure nodal planes under USWF exposure and further increase the binding opportunity. To mitigate electrostatic repulsion and facilitate binding strength during ultrasonic exposure, cationic polyethyleneimine (PEI) was complexed with plasmid DNA. The USWF in a tubular chamber was generated from the interference of acoustic waves launched from the bottom piezoelectric transmitter and reflected by the top glass coverslip. Over the period of USWF exposure, K562 erythroleukemia cells arrived at the pressure nodal planes first and formed cell bands. The PEI/DNA complexes then used the preformed cell bands as the nucleating sites on which to attach. As a result, the collision probability of PEI/DNA complexes and target cells was increased and consequently led to the augmentation of transfection efficiency.

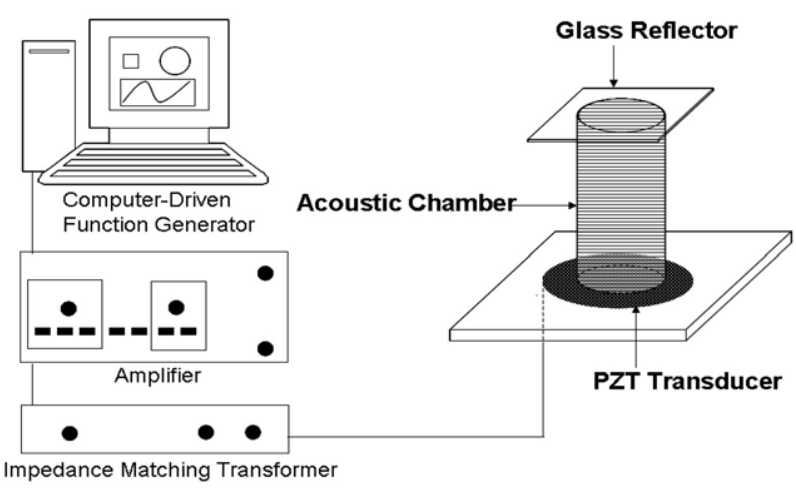

Fig. 1. Schematic diagram of experimental set-up. The lead zirconate titanate piezoelectric transducer, bonded on a plastic platform, was connected to an impedance matching transformer that was serially connected with an amplifier and a computerdriven function generator. After being filled with culture medium containing cells and/or PEI/DNA complexes, the tubular acoustic chamber was placed on the PZT transducer smeared with glycerol as the coupling agent. The acoustic waves, transmitted from the transducer at $1 \mathrm{MHz}$ and $25 \mathrm{~V}_{\mathrm{p}-\mathrm{p}}$, were reflected from the glass coverslip capped on the top of the chamber. The interference of transmitted and reflected waves led to the formation of ultrasound standing wave fields. 


\section{MATERIALS AND METHODS}

\section{Acoustic set-up}

The 1-MHz acoustic resonance with $25 \mathrm{~V}_{\mathrm{p}-\mathrm{p}}$ (peakto-peak voltage) of energy amplitude was generated from the interference of waves transmitted from the piezoelectric transducer on the bottom of the acoustic chamber and reflected from the top glass coverslip (Fig. 1). The piezoelectric transducer was made of a $25-\mathrm{mm}$ diameter and 2-mm thick lead zirconate titanate (PZT) disc (PZT5800; Channel Industries Inc., Santa Barbara, CA, USA), which was bonded on a plastic platform to transmit ultrasonic waves into the acoustic chamber placed on top of it. Before energizing the PZT transducer, the sinusoidal wave generated from a computer-driven 16$\mathrm{MHz}$ function generator card (NI PCI-5401; National Instruments Corp., Austin, TX, USA) was augmented by an amplifier (model 7500; Krohn-Hite Corp., Brockton, MA, USA) connected with an impedance-matching transformer (MT-56; Krohn-Hite Corp.). The tubular acoustic chamber, made of plexiglas, was fabricated with the dimensions of 13-mm inner diameter, 38- $\mathrm{mm}$ height and $0.5-\mathrm{mm}$ thickness. The chamber, filled with the medium containing cells and/or DNA vectors and covered with a borosilicate glass reflector $(22 \mathrm{~mm} \times 22 \mathrm{~mm} \times$ $0.15 \mathrm{~mm}$ ), was laid on the PZT diskette, which was pre-smeared with glycerol as the coupling agent.

\section{Cell culture and cell viability after USWF exposure}

Human erythroleukemia K562 cells (ATCC; American Type Culture Collection, Rockville, MD, USA) were grown in a spinner flask with Dulbecco's Modified Eagle Medium (Irvine Scientific, Santa Ana, CA, USA) supplemented with $10 \%$ fetal bovine serum (Gibco BRL, Rockville, MD, USA) (D10 medium in abbreviation) and $100 \mathrm{U} / \mathrm{mL}$ penicillin/streptomycin (Irvine Scientific). Cells were cultivated in a $5 \% \quad \mathrm{CO}_{2}$ atmosphere and balanced with $100 \%$ humidity at $37^{\circ} \mathrm{C}$.

To assess the influence of USWF on cells, cell viabilities after 0 -h and 24-h USWF exposure were determined by hemocytometry with trypan-blue staining.

\section{Preparation of plasmid DNA}

The enhanced green fluorescent protein (EGFP)encoding plasmid DNA used in this study was pEGFP-C3 (4.7 kb; Clontech, Palo Alto, CA, USA). The plasmid DNA was amplified in DH5 $\alpha$-competent cells and isolated from the bacteria using Qiagen mini kit-25 (Qiagen, Valencia, CA, USA). Briefly, the bacterial cells were harvested and lysed in the $\mathrm{NaOH} / \mathrm{SDS}$ buffer with 0.1 wt \% RNase and then neutralized by potassium acetate. After separating nonsoluble precipitated debris, the clear lysate was loaded to the Qiagen-tips containing anion-exchange resin, followed by washing with buffer to remove the remains. The plasmid DNA was eluted with buffer containing $1.25 \mathrm{M} \mathrm{NaCl}$ and then desalted and precipitated using isopropanol. The plasmid DNA precipitate was centrifuged for $30 \mathrm{~min}$ and washed twice with $70 \%$ ethanol to replace isopropanol. After centrifugation, the plasmid DNA was air dried and dissolved in Tris-EDTA buffer for $-20^{\circ} \mathrm{C}$ storage. The concentration of isolated plasmid DNA was determined by measuring absorption at 260-nm wavelength $\left(\mathrm{A}_{260}\right)$ using UV spectrophotometry (DU-640; Beckman Coulter, Inc., Chaska, MN, USA). In addition, the absorbance ratio of the $A_{260}$ to $\mathrm{A}_{280}$ was between 1.7 and 2.0, indicating that the purified plasmid DNA was free of contaminants.

\section{Preparation of PEI/DNA complexes}

DNA complexation was performed by mixing PEI $(\mathrm{MW}=750 \mathrm{kDa}$; Sigma, St. Louis, MO, USA) and plasmid DNA at 6:1 of N/P ratio in the $\mathrm{NaCl}$ aqueous solution. Briefly, $4 \mu \mathrm{g}$ of plasmid DNA and the desired amount of PEI were separately dissolved in $100 \mu \mathrm{L} \mathrm{NaCl}$ aqueous solutions $(150 \mathrm{mM})$. Then, the solution containing PEI was added to the medium containing plasmid DNA. After gently mixing for $1 \mathrm{~min}$, the solution was incubated at room temperature for $30 \mathrm{~min}$ and the PEI/ DNA complexes were formed. The size of DNA complexes was measured by the dynamic light scattering (DynaPro-99EMS/X; Wyatt Technology Corp., Santa Barbara, CA, USA).

\section{Integrity of plasmid DNA post-USWF exposure}

The DNA integrity after USWF exposure was examined by gel electrophoresis. After 5-min ultrasonic irradiation, $2 \mu \mathrm{g}$ of plasmid DNA in $5 \mathrm{~mL}$ of D10 medium was harvested by centrifugation for $30 \mathrm{~min}$ at $15,100 \times g$ twice and resuspended in $12 \mu \mathrm{L}$ Tris-EDTA buffer. Samples, including plasmid DNA with or without USWF exposure, were loaded onto 0.7 wt $\%$ of agarose gel and electrophorized for $90 \mathrm{~min}$ at $115 \mathrm{~V}$. The separations were visualized by ethidium bromide staining under UV illumination.

\section{Nonviral transfection under USWF exposure}

A total of $10^{7} \mathrm{~K} 562$ cells in the exponential growth phase were harvested and aliquotted into two acoustic chambers with $5 \mathrm{~mL}$ of D10 medium for each. PEI/DNA complexes were then added into both chambers and one of them was exposed to $1-\mathrm{MHz}$ USWF for $5 \mathrm{~min}$. After ultrasonic exposure, cells of both sets were incubated for $4 \mathrm{~h}$ at $37^{\circ} \mathrm{C}$ and then centrifuged and resuspended into two T-flasks. After additional 48-h cultivation, DNA transfection efficiencies were determined by detecting the intensity of EGFP transgene expression and scoring the number of transfected K562 cells. The fluorescent intensity of EGFP-expressing cells represented by rela- 


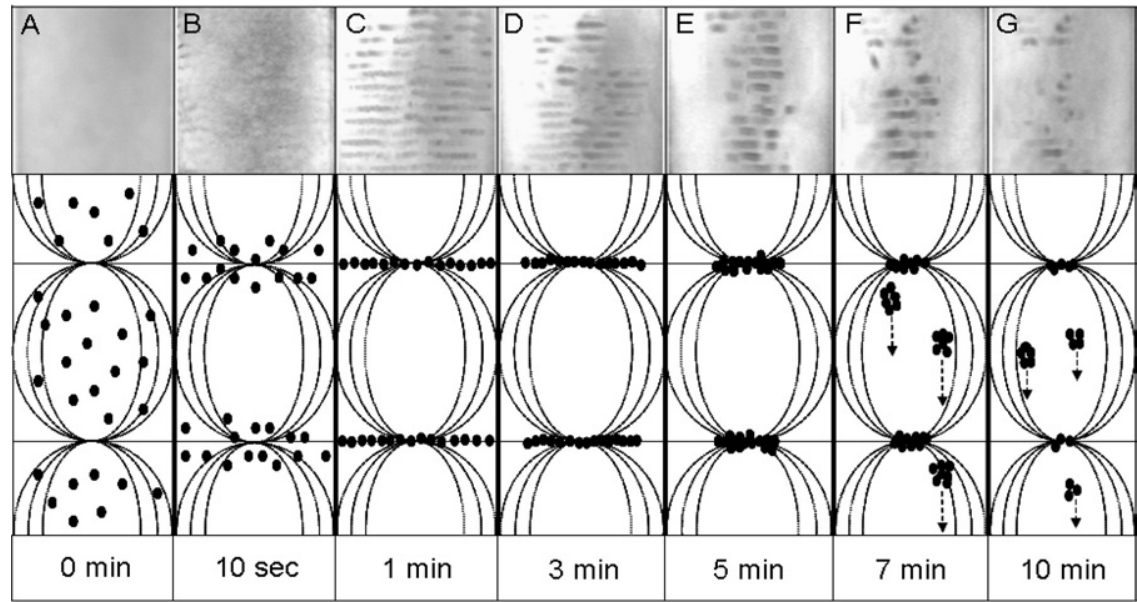

Fig. 2. The evolution of K562 cells in the USWF-generated chamber up to $10 \mathrm{~min}$ of acoustic exposure. Images were taken by a digital camera placed in front of the tubular acoustic chamber. The cell bands shown as dark stripes were separated by $750 \mu \mathrm{m}$ (i.e., half-wavelength of 1-MHz sound in water). The width of each photograph matches the diameter of the tubular chamber, which is $14 \mathrm{~mm}$. Total cell number was $5 \times 10^{6}$ suspended in 5 -mL culture medium. Schematic drawing represents the movement of K562 cells $(\bullet)$ under USWF exposure. Driven by the primary radiation force, cells were assembled on the pressure nodal planes in USWF and then aggregated to form cell bands with intervals of half-wavelength (A-C). Because of activation of the secondary radiation force, cells integrated tightly to each other; hence the slender cell bands were compressed and turned into short and thickening form (D and E). Once the body force of gradually enlarged cell agglomerates surpassed the acoustic radiation force, cell clumps collapsed, thereby resulting in the destruction of cell bands (F and $\mathrm{G})$.

tive fluorescence units (RFUs) was measured by a fluorometric microplate reader (SpectraMax M2; Molecular Devices Corp. Sunnyvale, CA, USA) set with $472 \mathrm{~nm}$ and $512 \mathrm{~nm}$ of excitation and emission wavelength, respectively. Before measuring RFUs, all samples were transferred to a six-well culture plate with an equal cell number of $10^{6}$ cells per well. In addition, photomicrographic images containing EGFP-expressing K562 cells were taken under both bright-field and fluorescent modes when operating fluorescent microscopy. The mean transfection percentage was calculated by counting total and transfected cells in 10 bright-field and fluorescent microscopic zones for each group.

\section{Nonviral transfection under ultrasonic traveling wave fields}

To distinguish the effect of USWF from sonoporation on nonviral transfection, three additional test groups were run that each contained $5 \mathrm{~mL}$ of cell supernatant $\left(5 \times 10^{6}\right.$ cells plus PEI/DNA complexes [N/P ratio $=$ 6:1]): a control with cells and PEI/DNA vectors only (A), those exposed to ultrasonic traveling wave fields (UTWF) (B) and those exposed to USWF (C). For (B), the USWF setup was modified by placing a silicon mat served as an acoustic absorber to minimize acoustic reflection and concomitant standing waves formation. After sonication of groups (B) and (C) with 1-MHz and $25-\mathrm{V}_{\mathrm{p}-\mathrm{p}}$ acoustic wave for $5 \mathrm{~min}$, all cells were incubated at $37^{\circ} \mathrm{C}$ for $4 \mathrm{~h}$ and then transferred into 10 -mL T-flasks and cultivated for another $48 \mathrm{~h}$. The intensities of EGFP expressed in K562 cells were measured using the fluorometric method mentioned above.

\section{Statistical analysis}

All of the experimental data were obtained in triplicate and presented as mean \pm standard error of the mean. Statistical comparison by the analysis of variance (ANOVA) was performed at a significant level of $p<$ 0.05 based on the Student's $t$-test.

\section{RESULTS}

\section{Cell band formation in USWF}

As shown in Fig. 2A-G, photographic images illustrate the formation and evolution of cell bands under USWF exposure at $1 \mathrm{MHz}$. In the first $5 \mathrm{~min}$ (A-E), suspended cells driven by the primary radiation force moved to the pressure nodal planes and formed bands perpendicular to the propagating direction of acoustic waves. Each band was separated in the interval of halfwavelength $(\sim 750 \mu \mathrm{m})$. During this period, the longer that the cells were exposed to USWF, the more distinct striated cell bands were observed. It is also clearly illustrated that cell clusters were contracted and concentrated in the central area of the chamber as a result of the generated lateral radiation force (Spengler et al. 2003). 
As time progressed over 5 min (shown in F and G), the body force of gradually enlarged cell agglomerates surpassed the acoustic radiation force thereby resulted in the sedimentation. Most of the cell bands became disturbed after $7 \mathrm{~min}$ of USWF exposure. Only a few short and discrete bands were left after 10 min of USWF exposure.

\section{K562 cell viability under USWF exposure}

To assess the bioeffect of USWF on K562 cells, cell viabilities were determined after cells were treated with USWF for different periods. The influence of USWF irradiation on cell viability under various exposure times was shown in Fig. 3. The two bars at each exposure time point represent the viabilities determined at $0 \mathrm{~h}$ and $24 \mathrm{~h}$ post-USWF exposure, respectively. Generally speaking, the viabilities were all $>90 \%$, although they were exposed to USWF for as long as $10 \mathrm{~min}$.

\section{Size of PEI/DNA complex and integrity of plasmid DNA under USWF exposure}

The size distribution of PEI/DNA complexes was measured by dynamic light scattering. Results showed that approximately $60 \%$ of PEI/DNA complexes were in the average size of $80 \mathrm{~nm}$ (data not shown). In addition, to determine the structural integrity of plasmid DNA after USWF exposure, gel electrophoresis analysis was performed. From the electrophoretic patterns of plasmid DNA shown in Fig. 4, the migration of plasmid DNA exposed to USWF (lane C) was similar to the one without ultrasonic treatment (lane B). In addition, although few relaxed (linear and open circular) plasmid DNA patterns were visible in lane $\mathrm{C}$, most of them remained in supercoiled form.

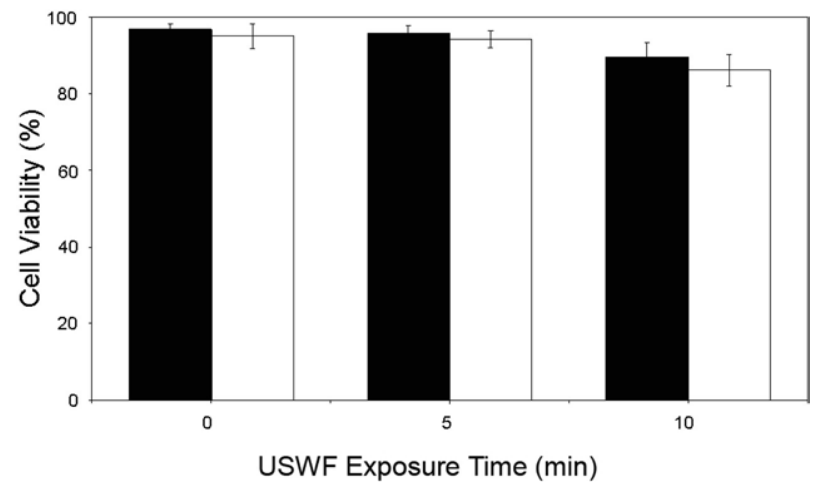

Fig. 3. Viability of K562 cells for various periods of USWF exposure. After ultrasonic irradiation for 0,5 and $10 \mathrm{~min}$, cell viabilities were counted immediately (ם) and 24-h postincubation ( $\square$ ), using a hemocytometer with trypan blue staining. Each bar represents the mean of three independent experiments. Error bars are standard error of the mean cell viability $(p<0.05)$.

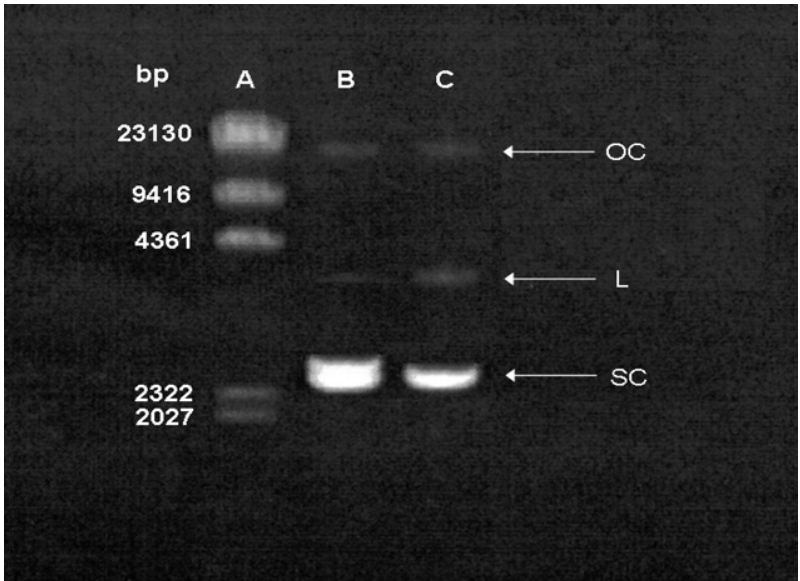

Fig. 4. Effect of acoustic pressure on plasmid DNA integrity. lane A: DNA marker; lane B: plasmid DNA $(1 \mu \mathrm{g})$ without USWF exposure; lane C: plasmid DNA $(1 \mu \mathrm{g})$ with USWF exposure for 5 min. OC: open circular; L: linear; SC: supercoiled.

Transfection of PEI/DNA complexes under USWF exposure

With the absence of a large change in cell viability and DNA integrity after USWF exposure, DNA transfection efficiency was examined using suspended K562 cells $\left(10^{6}\right.$ cells $\left./ \mathrm{mL}\right)$ and PEI/DNA complexes. According to the results illustrated in Fig. 2, cell bands remained intact after 5-min USWF exposure. Therefore, this operation time was selected for gene transfection, with the

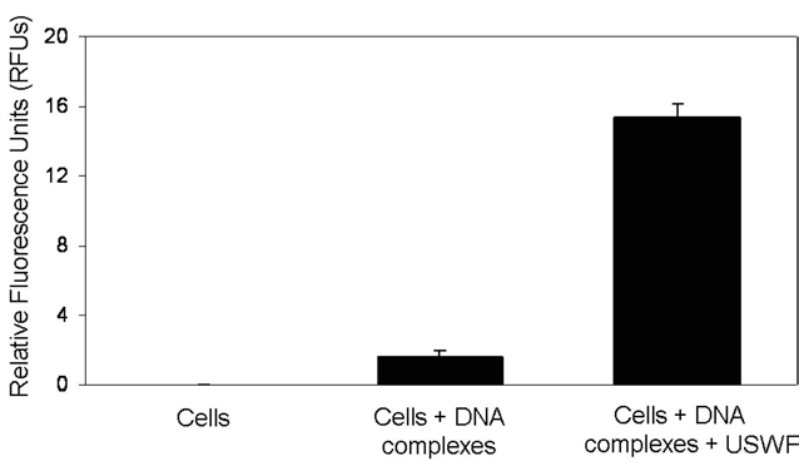

Fig. 5. Effect of USWF exposure on nonviral transfection efficiency. K562 cells were transfected with PEI/DNA complexes in the absence or presence of USWF exposure performed at $1 \mathrm{MHz}$ and $25 \mathrm{~V}_{\mathrm{p}-\mathrm{p}}$ for $5 \mathrm{~min}$. After additional 48-h incubation at $37^{\circ} \mathrm{C}$, transfection rates were measured by detecting fluorescence intensity using a fluorometric microplate reader set with $472 \mathrm{~nm}$ and $512 \mathrm{~nm}$ of excitation and emission wavelength, respectively. The corresponding values of relative fluorescence units (RFUs) for three groups (control, cells/DNA complexes with and without USWF exposure) were $0,1.62$ and 15.37, respectively. Error bars are the standard error of the mean RFUs obtained from three independent experiments $(p<0.05)$. 
(a)
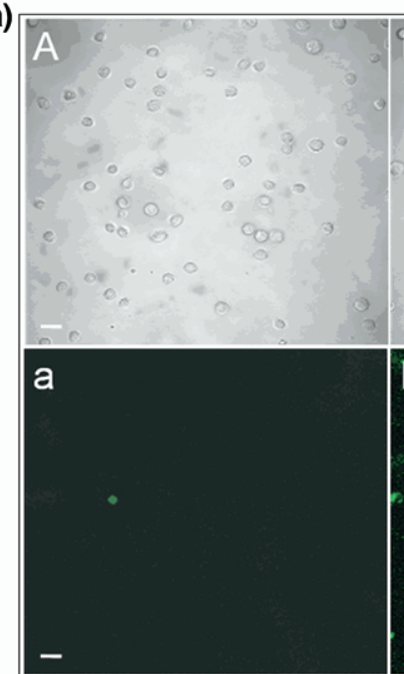

Without USWF Exposure
B

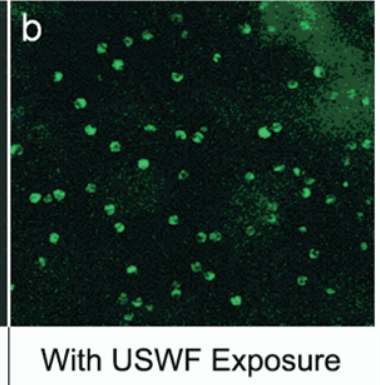

(b)

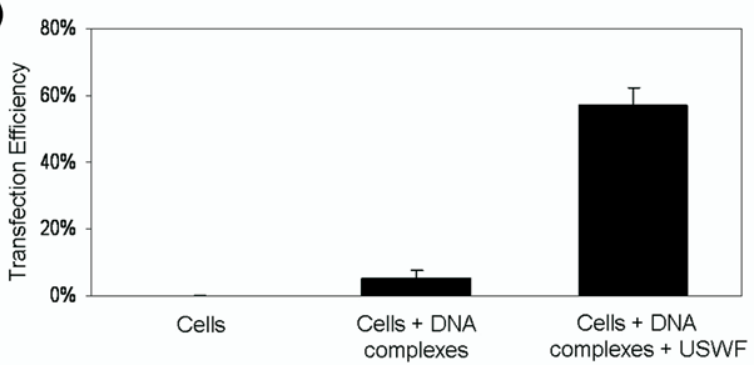

Fig. 6. (a) Photomicrographic images of K562 cells with EGFP expression. K562 cells were transfected with PEI/DNA complexes in the absence (A-a) or presence (B-b) of USWF exposure performed at $1 \mathrm{MHz}$ and $25 \mathrm{~V}_{\mathrm{p}-\mathrm{p}}$ for $5 \mathrm{~min}$. After additional 48 -h incubation at $37^{\circ} \mathrm{C}$, images of cells were taken by bright-field (A and B) and fluorescent microscopy ( $\mathrm{a}$ and b), respectively. Scale bar $=30 \mu \mathrm{m}$. (b) Transfection percentages of K562 cells with or without USWF exposure. Transfection efficiencies were measured, based on the ratio of K562 cells with EGFP expression to the total cell number enumerated in 10 bright-field microscopic zones. The percentages of EGFPexpressing cells detected by fluorescent microscopy were calculated to be $0 \%, 5 \%$ and $57 \%$, respectively. Error bars are the standard error of the mean transfection percentage obtained from 10 independent photomicrographic images for each set $(p<0.05)$.

anticipation that PEI/DNA complexes will have more chance to encounter preformed cell bands that were in a relatively undisturbed state. After $48 \mathrm{~h}$ of incubation at $37^{\circ} \mathrm{C}$ after USWF exposure, nonviral transfection efficiency was measured, based on EGFP transgene expression using fluorometry and fluorescent microscopy. Figure 5 shows the intensity of EGFP expression in the transfected K562 cells detected by a fluorometric microplate reader. The level of EGFP intensity with USWF treatment increased approximately 10 -fold in compari-

son with the one without USWF exposure. The photomicrographic images shown in Fig. 6(a) further corroborate the fluorometric results given in Fig. 5. The mean transfection percentages were further calculated according to 10 photomicrographic images and giving an $\sim 11$-fold increment (from 5\% to 57\%), as shown in Fig. 6(b).

\section{$U T W F$ vs. $U S W F$}

The level of EGFP expression in K562 cells was determined using UTWF, where cells and PEI/DNA complexes remained homogeneously distributed in the culture medium and were continuously applied to high local pressure amplitudes during exposure. Figure 7 shows that the EGFP intensity obtained after 5-min UTWF exposure was almost the same as the one without ultrasonic treatment. However, there was an approximately 10-fold increment of EGFP intensity using USWF.

\section{DISCUSSION}

Since 1987, when ultrasound was first reported as a feasible method for nonviral gene delivery (Fechheimer et al. 1987), ultrasound-driven inertial cavitation has been extensively developed in the past two decades. As a previously unexplored physical force used for nonviral gene delivery, megahertz ultrasound standing wave fields were explored in this study to see whether nonviral transfection efficiency can be augmented or not. It was shown that suspended K562 cells under USWF exposure could move to the pressure nodal planes and form cell

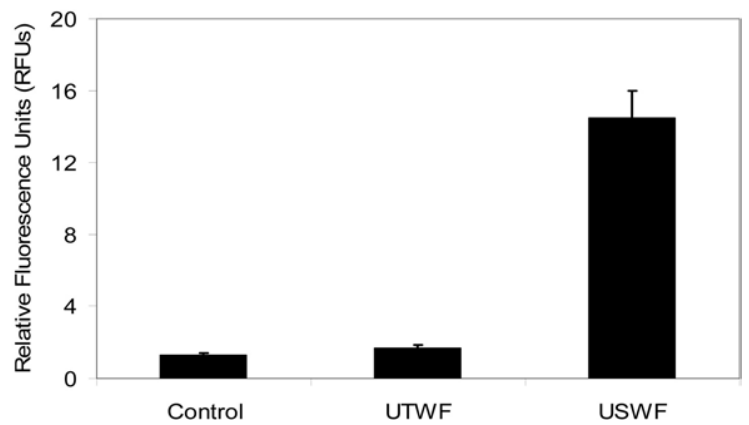

Fig. 7. Transfection efficiency under USWF and UTWF exposure. K562 cells were transfected by nonviral vectors under ultrasonic exposure (i.e., USWF or UTWF) or not. For the control, cells were transfected with PEI/DNA complexes alone; for USWF, cells were transfected with PEI/DNA complexes at $1 \mathrm{MHz}$ and $25 \mathrm{~V}_{\mathrm{p} \text {-p }}$ USWF for $5 \mathrm{~min}$; for UTWF, cells were transfected with PEI/ DNA complexes under UTWF exposure using the same operation parameters as for the USWF setting. The intensities of EGFP expressed in K562 cells were measured after 48-h incubation using the fluorometric method. Error bars are the standard error of the mean relative fluorescence units (RFUs) obtained from three independent experiments $(p<0.05)$. 
bands by the primary radiation force. Then, nanometersized PEI/DNA complexes $(\sim 80 \mathrm{~nm})$ could be trapped by the striated cell bands when they circulated through the porous network formed by the cell agglomerates. One order of magnitude increase of nonviral transfection efficiency was obtained using USWF operation (shown in Figs. 5 and 6(b). However, the pertinent question is whether the increment of transfection efficiency was partly attributed to sonoporation. To clarify that the enhanced nonviral transfection was mainly attributed to USWF rather than to sonoporation generated by tiny acoustic cavitation, the level of EGFP expression in K562 cells was determined using UTWF. Because the ultrasonic intensity applied to USWF and UTWF systems was the same (i.e., $25 \mathrm{~V}_{\mathrm{p}-\mathrm{p}}$ ), the degree of sonoporation, if there was any, had to be identical in both acoustic fields. Therefore, if sonoporation plays a dominant role in enhancing nonviral transfection efficiency, the latter should have a similar intensity of EGFP expression in cells as the former. As shown in Fig. 7, the EGFP intensity of cells exposed to UTWF was close to the one without ultrasonic treatment, but 10-fold less than the one exposed to USWF. Because the prominent difference between UTWF and USWF is that cells form bands in the latter and not in the former, it can therefore be concluded that the enhancement of nonviral transfection resulted from the increased collision probability of cells and PEI/DNA complexes, instead of from the effect of sonoporation.

Under acoustic exposure, shear stress generated by acoustic microstreaming might affect cell physiology and, therefore, result in apoptosis. To clarify this concern, cell viability after USWF exposure was examined. As shown in Fig. 3, >90\% of cells remained viable within 10-min USWF exposure. In addition, USWFtreated cells were cultivated at $37^{\circ} \mathrm{C}$ for 5 consecutive days and revealed a similar growth kinetic profile as the cells without USWF exposure (data not shown). This indicates that the effect of USWF on the physiologic state of suspended cells was insignificant compared with exposure under cavitation field (Guzman et al. 2001). It is probably the result of cells moving quickly to the pressure nodes where the primary radiation force is the minimum (theoretically it is zero) thereby diminishing the harmful bioeffects from USWF exposure. This result is consistent with a multitude of studies (Doblhoff-Dier et al. 1994; Radel et al. 2000; Gaida et al. 1996).

It is obvious that USWF exposure time plays an important role in the encounters of cells and DNA complexes. That is, the longer the exposure period, the more binding was that the opportunity attained between cells and nonviral DNA vectors. However, driven by the primary radiation force, cells were agglomerated and formed bands in a short time frame, then fell down as time progressed further, as a result of gravitation imposed on gradually enlarged agglomerates. Apparently, DNA complexes will not have cell bands on which to attach to if cells, precipitated down to the bottom of the acoustic chamber. Hence, to optimize the period of USWF exposure, the time required to sustain cell bands and, consequently, increase cell-vector encounters in the acoustic chamber, is the essential operating parameter needing to be determined. From our observation shown in Fig. 2, within 5-min ultrasonic irradiation, cell bands remained persistently in the USWF, although they were contracted and became thicker by the secondary radiation force (Spengler et al. 2003). However, 7 min after USWF exposure, cell bands started to collapse, as a result of gravitation and lost capability of trapping DNA vectors. As a result, we picked $5 \mathrm{~min}$ as the optimal USWF exposure time for the nonviral transfection under $1 \mathrm{MHz}$ using $10^{6}$ cells $/ \mathrm{mL}$.

Because of ultrasonic stresses, the integrity and stability of plasmid DNA may be altered, thereby leading to detrimental effects on the efficacy of gene transfer and transgene expression (Walther et al. 2003). To assess the influence of USWF exposure on plasmid DNA, agarose gel electrophoresis was performed. By comparing the electrophoretic patterns between plasmid DNA with and without 5-min ultrasonic treatment, the structure change could be identified indirectly. As shown in Fig. 4, although few relaxed (linear and open circular) plasmid DNA patterns were visible in lane $\mathrm{C}$, most of them remained in supercoiled form, indicated that there was insignificant effect on naked plasmid DNA after USWF exposure. Furthermore, from reported studies (Kircheis et al. 2001; Ogris et al. 1998), negatively charged DNA could be protected from degradation by electrostatic interaction with condensing compound (e.g., polycationic PEI) during mechanical process; therefore, the quality and conformation of plasmid DNA after USWF exposure were assured.

In addition to preventing plasmid DNA from degradation under USWF exposure, PEI offering positivelycharged shielding played an important role on the transfection process. If only naked DNA plasmids were treated in USWF with K562 cells, the nonviral transfection rate was $<5 \%$ (data not shown). However, 57\% of transfection efficiency was obtained in the presence of PEI (i.e., PEI/ DNA complexes) under USWF exposure. This is because the electrostatic attraction between positive PEI/DNA complexes and anionic components on the cell membrane (e.g., proteoglycans) can resist the microstreaming drags once PEI/DNA complexes move into the vicinity of the target cell surface and further facilitate cellular uptake.

For large-scale ultrasound standing wave fields, bulk-type Eckart acoustic streaming, different from the aforementioned microstreaming, is the main challenge to overcome. This acoustic streaming could be eliminated 
in a short container (Spengler and Jekel 2000); however, it arises because of energy transfer mechanisms in the bulk phase as a result of energy absorption in the fluid and dissipation at the interface between the fluid and chamber surface (Shi et al. 2002; Zauhar et al. 1998). Eckart streaming may drive suspended cells out of desired positions or even disrupt cell collections on the pressure nodal planes. To maintain the pressure amplitude and sustain good cell suspension during large-scale USWF exposure, acoustically transparent films can be deployed perpendicular to the direction of ultrasound waves, thereby dividing the large-scale chamber into domains of shorter free path-length, which could reduce the bulk streaming velocity and retain cell band formation on the pressure nodal planes.

\section{SUMMARY}

In this study, USWF was demonstrated to be a feasible approach to enhance the efficiency of nonviral transfection. Under USWF exposure, cells can agglomerate on the pressure nodal planes and circumvent severe damage from ultrasonic irradiation. In addition, nanometer-sized PEI/DNA complexes could be brought to cell clumps by microstreaming drag force, thereby facilitating the process of gene delivery.

Acknowledgments-This work was supported in part by grant R21 EB04117 from the National Institutes of Health and in part by the National Taiwan University.

\section{REFERENCES}

Bao S, Thrall BD, Miller DL. Transfection of a reporter plasmid into cultured cells by sonoporation in vitro. Ultrasound Med Biol 1997; 23:953-959.

Chen SY, Shohet RV, Bekeredjian R, Frenkel P, Grayburn PA. Optimization of ultrasound parameters for cardiac gene delivery of adenoviral or plasmid deoxyribonucleic acid by ultrasound-targeted microbubble destruction. J Am Coll Cardiol 2003;42:301-308.

Coakley WT, Bardsley DW, Grundy MA. Cell manipulation in ultrasonic standing wave fields. J Chem Tech Biotechnol 1989;44: 43-62.

Doblhoff-Dier O, Gaida T, Katinger H, Burger W, Groschl M, Benes E. A novel ultrasonic resonance field device for the retention of animal cells. Biotechnol Prog 1994;10:428-432.

Fechheimer M, Boylan JF, Parker S, Sisken JE, Patel GL, Zimmer SG. Transfection of mammalian cells with plasmid DNA by scrape loading and sonication loading. Proc Natl Acad Sci U S A 1987; 84:8463-8467.

Gaida T, Doblhoff-Dier O, Strutzenberger K, Katinger H, Burger W, Groschl M, Handl B, Benes E. Selective retention of viable cells in ultrasonic resonance field devices. Biotechnol Prog 1996;12:73-76.

Guzman HR, Nguyen DX, Khan S, Prausnitz MR. Ultrasound-mediated disruption of cell membrane. I. Quantification of molecular uptake and cell viability. J Acoust Soc Am 2001;110:588-596.

Hacein-Bey-Abina S, Von Kalle C, Schmidt M, McCormack MP, Wulffraat N, Leboulch P, Lim A, Osborne CS, Pawliuk R, Morillon E, Sorensen R, Forster A, Fraser P, Cohen JI, de Saint Basile G, Alexander I, Wintergerst U, Frebourg T, Aurias A, Stoppa-Lyonnet D, Romana S, Radford-Weiss I, Gross F, Valensi F, Delabesse E, Macintyre E, Sigaux F, Soulier J, Leiva LE, Wissler M, Prinz C, Rabbitts TH, Le Deist F, Fischer A, Cavazzana-Calvo M. LMO2- associated clonal $\mathrm{T}$ cell proliferation in two patients after gene therapy for SCID-X1. Science 2003;302:415-419.

Hawkes JJ, Barrow D, Cefai J, Coakley WT. A laminar flow expansion chamber facilitating downstream manipulation of particles concentrated using an ultrasonic standing wave. Ultrasonics 1998;36:901903.

Huber PE, Jenne J, Debus J, Wannenmacher MF, Pfisterer P. A comparison of shock wave and sinusoidal-focused ultrasound-induced localized of HeLa cells. Ultrasound Med Biol 1999;25:14511457.

Joersbo M, Brunstedt J. Sonication: A new method for gene transfer to plants. Physiol Plant 1992;85:230-234.

Kim HJ, Greenleaf JF, Kinnick PR, Bronk JT, Bolander ME. Ultrasound-mediated transfection of mammalian cells. Human Gene Ther 1996;7:1339-1346.

Kircheis R, Wightman L, Wagner E. Design and gene delivery activity of modified polyethyleneimines. Adv Drug Del Rev 2001;53: 341-358.

Lauer U, Burgelt E, Squire Z, Messmer K, Hofschneider PH, Gregor M, Delius M. Shock wave permeabilization as a new gene transfer method. Gene Ther 1997;4:710-715.

Lawrie A, Brisken AF, Francis SE, Cumberland DC, Crossman DC, Newman CM. Microbubble-enhanced ultrasound for vascular gene delivery. Gene Ther 2000;7:2023-2027.

Lee Y-H, Peng C-A. Enhanced retroviral gene delivery in ultrasonic standing wave fields. Gene Ther 2005;12:625-633.

Lee Y-H, You J-O, Peng C-A. Retroviral transduction of adherent cells in resonant acoustic fields. Biotech Progress 2005;21:372-376.

Limaye MS, Hawkes JJ, Coakley WT. Ultrasonic standing wave removal of microorganisms from suspension in small batch systems. J Microbiol Methods 1996;27:211-220.

Lu QL, Liang HD, Partridge T, Blomley MJK. Microbubble ultrasound improves the efficiency of gene transduction in skeletal muscle in vivo with reduced tissue damage. Gene Ther 2003;10:396-405.

Luo D, Saltzman WM. Synthetic DNA delivery systems. Nature Biotechnol 2000;18:33-37.

Marshall E. Gene therapy death prompts review of adenovirus vector. Science 1999;286:2244-2245.

Miller DL. A review of the ultrasonic bioeffects of microsonation, gas-body activation, and related cavitation-like phenomena. Ultrasound Med Biol 1987;13:443-470.

Miller DL, Bao S, Gies RA, Thrall BD. Ultrasonic enhancement of gene transfection in murine melanoma tumors. Ultrasound Med Biol 1999;25:1425-1430.

Miller DL, Pislaru SV, Greenleaf JE. Sonoporation: Mechanical DNA delivery by ultrasonic cavitation. Somat Cell Mol Genet 2002;27: $115-134$.

Miller DL, Song J. Tumor growth reduction and DNA transfer by cavitation-enhanced high-intensity focused ultrasound in vivo. Ultrasound Med Biol 2003;29:887-893.

Ogris M, Steinlein P, Kursa M, Mechtler K, Kircheis R, Wagner E. The size of DNA/transferrin-PEI complexes is an important factor for gene expression in cultured cells. Gene Ther 1998;5:1425-1433.

Pislaru SV, Pislaru C, Kinnick RR, Singh R, Gulati R, Greenleaf JF, Simari RD. Optimization of ultrasound-mediated gene transfer: Comparison of contrast agents and ultrasound modalities. Eur Heart J 2003;24:1690-1698.

Radel S, McLoughlin AJ, Gherardini L, Doblhoff-Dier O, Benes E. Viability of yeast cells in well controlled propagating and standing ultrasonic plane waves. Ultrasonics 2000;38:633-637.

Shi XG, Martin RW, Vaezy S, Crum LA. Quantitative investigation of acoustic streaming in blood. J Acoust Soc Am 2002;111:11101121.

Shirgaonkar IZ, Lanthier S, Kamen A. Acoustic cell filter: A proven cell retention technology for perfusion of animal cell cultures. Biotechnol Adv 2004;22:433-444.

Spengler JF, Coakley WT, Christensen KT. Microstreaming effects on particle concentration in an ultrasonic standing wave. AIChE J 2003;49:2773-2782. 
Spengler J, Jekel M. Ultrasound conditioning of suspensions-Studies of streaming influence on particle aggregation on a lab-and pilotplant scale. Ultrasonics 2000;38:624-628.

Taniyama Y, Tachibana K, Hiraoka K, Aoki M, Yamamoto S, Matsumoto K, Nakamura T, Ogihara T, Kaneda Y, Morishita R. Development of safe and efficient novel nonviral gene transfer using ultrasound: Enhancement of transfection efficiency of naked plasmid DNA in skeletal muscle. Gene Ther 2002;9:372-380.

Tata DB, Dunn F, Tindall DJ. Selective clinical ultrasound signals mediate differential gene transfer and expression in two human prostate cancer cell lines: LnCap and PC-3. Biochem Biophys Res Commun 1997;234:64-67.

Whitworth G, Coakley WT. Particle column formation in a stationary ultrasonic field. J Acoust Soc Am 1992;91:79-85.

Walther W, Stein U, Voss C, Schmidt T, Schleef M, Schlag PM Stability analysis for long-term storage of naked DNA: Impact on nonviral in vivo gene transfer. Analytical Biochemistry 2003;318:230-235.

Zauhar G, Starritt H, Duck F. Studies of acoustic streaming in biological fluids with an ultrasound Doppler technique. Br F Radiol 1998;71:297-302. 